\title{
Modeling Magnetospheric Sources
}

\author{
Raymond J. Walker ${ }^{1,2}$ \\ Maha Ashour-Abdalla ${ }^{1,3}$ \\ Tatsuki Ogino 4 \\ Vahe Peroomian ${ }^{1}$ \\ Robert L. Richard ${ }^{1}$
}

UCLA Institute of Geophysics and Planetary Physics Preprint 5678

September 21, 2001

\footnotetext{
${ }^{1}$ Institute of Geophysics and Planetary Physics, University of California, Los Angeles, CA, 90095-1567

${ }^{2}$ Department of Earth and Space Sciences, University of California, Los Angeles, CA, 90095-1567

${ }^{3}$ Department of Physics and Astronomy, University of California, Los Angeles, CA, 90095-1547

${ }^{4}$ Solar Terrestrial Environment Laboratory, Nagoya University, Toyokawa, Aichi, Japan
} 


\begin{abstract}
We have used global magnetohydrodynamic simulations of the interaction between the solar wind and magnetosphere together with single particle trajectory calculations to investigate the sources of plasma entering the magnetosphere. In all of our calculations solar wind plasma primarily enters the magnetosphere when the field line on which it is convecting reconnects. When the interplanetary magnetic field has a northward component the reconnection is in the polar cusp region. In the simulations plasma in the low latitude boundary layer (LLBL) can be on either open or closed field lines. Open field lines occur when the high latitude reconnection occurs in only one cusp. In the MHD calculations the ionosphere does not contribute significantly to the LLBL for northward IMF. The particle trajectory calculations show that ions preferentially enter in the cusp region where they can be accelerated by non-adiabatic motion across the high latitude electric field. For southward IMF in the MHD simulations the plasma in the middle and inner magnetosphere comes from the inner (ionospheric) boundary of the simulation. Solar wind plasma on open field lines is confined to high latitudes and exits the tailward boundary of the simulation without reaching the plasma sheet. The LLBL is populated by both ionospheric and solar wind plasma. When the particle trajectories are included solar wind ions can enter the middle magnetosphere. We have used both the MHD simulations and the particle calculations to estimate source rates for the magnetosphere which are consistent with those inferred from observations.
\end{abstract}

\title{
1. Introduction
}

There are two main sources of magnetospheric plasma: the solar wind and the ionosphere. Classical theory of the solar wind interaction with the magnetosphere predicts that the magnetopause should be an impenetrable boundary separating the 
shocked solar wind plasma of the magnetosheath from the hot tenuous plasma of the magnetosphere. However, three decades of observations have demonstrated clearly that magnetosheath plasma exists inside all regions of the magnetopause (see Sibeck et al. [1999] for a recent review). These magnetospheric boundary layers include the lowlatitude boundary layer (LLBL), the polar cusp entry layer and the high latitude plasma mantle (PM).

Observations from low-altitude spacecraft indicate that shocked solar wind plasma from the magnetosheath enters the magnetosphere over a wide region under almost all solar wind conditions [e.g. Newell and Meng, 1992]. Observations in the magnetotail lobes indicate that plasma enters the magnetosphere along its entire length. Recently Hultqvist et al. [1999] have combined the results from a large number of studies to identify and quantify the major source regions for magnetospheric plasma. They estimate that $\sim 10^{26} \mathrm{~s}^{-1}$ solar wind particles enter through the dayside magnetopause while $10^{28}-10^{29} \mathrm{~s}^{-1}$ enters along the tail magnetopause. However most of the particles that enter through the tail magnetopause also exit via the tail and never reach the closed field line region of the magnetosphere where the required source strength is only a few times $10^{26} / \mathrm{s}$. Similarly, Moore et al. [1999] organized the published observations from polar orbiting spacecraft [Yau et al., 1985; Abe et al., 1996] to summarize the ionospheric outflow rates. Their results show that the ionosphere can provide $\sim 10^{26} / \mathrm{s}$ of $\mathrm{O}^{+}$and $\mathrm{H}^{+}$to the magnetosphere.

A number of processes have been proposed to account for the transfer of solar wind plasma into the magnetosphere. These include magnetic reconnection, finite Larmor radius effects, diffusion, the Kelvin-Helmholtz instability, impulsive penetration and 
direct cusp entry. In this paper we use computer simulations to study the sources of plasma for the magnetosphere with emphasis on the sources of the LLBL. First we investigate the sources of plasma by using a global magnetohydrodynamic (MHD) simulation of the interaction of the solar wind with the magnetosphere. We use the MHD model to investigate both the ionospheric and solar wind sources. Then we add single particle trajectory calculations of solar wind ions to the global MHD calculations in order to estimate the importance of phenomena such as finite Larmor radius effects not included in MHD. For each approach we estimate the source rate. In section 2 we briefly describe the models and techniques used in this study. The simulation results for northward IMF are presented in section 3 while those for southward IMF are in section 4 . Finally in section 5 we discuss these results in the context of magnetospheric observations.

\section{Approach}

Since the details of the global magnetohydrodynamic simulation code used for this study have been described previously [Ogino et al., 1992; 1994] we will describe it only briefly. We solve the resistive MHD equations and Maxwell's equations as an initial value problem on a rectangular grid. For the cases with purely northward and southward IMF we used a $(402 \times 102 \times 102)$ points grid with a uniform mesh size of $0.4 R_{E}$. For these calculations the simulation box extends from $30 R_{E} \geq x \geq-130 R_{E}, 0<y \leq-40 R_{E}$, and $0<z \leq 40 R_{E}$. We also have included results from simulations with $\operatorname{IMF} B_{Y} \neq 0$. For these cases we used a simulation box with $322 \times 82 \times 162$ points and a mesh size of $0.5 R_{E}$. In the $\mathrm{Z}$ direction the calculation domain is $-40 R_{E} \leq z \leq 40 R_{E}$. We solve the 
differential equations by using a modified version of the Leapfrog scheme that is a combination of the Leapfrog scheme and the two-step Lax-Wendroff scheme. The simulation parameters are fixed to solar wind values at the upstream edge of the simulation box with free boundary conditions at the sides and back. For all the simulations we used symmetry boundary conditions at $y=0$ while for the quarter magnetosphere calculation we also used symmetry boundary conditions at $z=0$. The ionospheric boundary is at $3.5 \mathrm{R}_{\mathrm{E}}$. The solar wind velocity was $300 \mathrm{~km} / \mathrm{s}$, the density was $5 \mathrm{~cm}^{-3}$ and the temperature was $2 \times 10^{50} \mathrm{~K}$.

The simulation for northward IMF was initialized by using an unmagnetized solar wind flow that lasted for up to three hours. Then a northward IMF was introduced into the flow. After four hours with northward IMF during which time a quasi-steady magnetosphere developed, the IMF was turned southward and the simulation was run for another four hours. For the simulations with $B_{\mathrm{Y}} \neq 0$ the initialization period was followed by a five-hour interval with northward IMF. Then the IMF was rotated in $15^{\circ}$ increments in the YZ plane with a dwell time at each direction of 15 minutes [Walker et al., 1999]. While the dwell time was sufficient to enable us to determine the entry mechanism, the overall magnetospheric configuration contains the effects of changing IMF orientations [Walker et al., 1999]. For selected IMF directions the dwell time was increased to 2 hours to enable us to investigate the configuration of a quasi-steady magnetosphere. For all of the simulations $|\vec{B}|=5 n T$.

We also examined the entry of solar wind ions into the magnetosphere by calculating the ion trajectories using a fixed magnetic and electric field model [AshourAbdalla et al., 1993]. We use magnetic and electric fields from the MHD simulations. 
The electric field is given by $\vec{E}=-\vec{v} \times \vec{B}+\eta \vec{J}$ where $\vec{v}$ is the velocity, $\vec{B}$ the magnetic field, and $\vec{J}$ is the current density. An explicit resistivity $(\eta)$ is included in the MHD simulations [Ogino et al., 1994]. In each case a distribution of ions with the temperature used in the MHD simulations was launched in the solar wind upstream of the bow shock. The ion trajectories were calculated by solving the equation of motion by using a fourth order Runge-Kutta method.

\section{Northward IMF Results}

We started our studies by modeling plasma entry when the interplanetary magnetic field was northward. In Figure 1 we have plotted flow streamlines for a case with purely northward IMF. We show one quarter of the magnetosphere with pressure contours on the noon-midnight meridian and equatorial planes. The bottom panel contains a blow up of the region near the Earth. This simulation was run for 3 hours with no IMF and then for 4 hours with a northward IMF. This snapshot was taken 2 hours after the northward IMF entered the simulation box and after a quasi-steady magnetospheric configuration had developed [Ogino et al., 1994; Bargatze et al., 1999]. Most of the flow impinging on the magnetosphere is diverted around the obstacle (top). However, some plasma can enter the magnetosphere. In the bottom panel we have plotted additional streamlines from the region around the subsolar point. These streamlines enter the magnetosphere and move tailward on magnetospheric field lines. In Figure 2 we have reproduced two of these flow streamlines and calculated magnetic field lines along each streamline. The red field lines correspond to a streamline along the noon-midnight meridian slightly northward of the equator. This streamline moves from solar wind to 
magnetospheric field lines at location 3 when the field line on which it is convecting reconnects in the polar cusp region forming a new closed magnetospheric field line. The streamline then moves around the flank magnetosphere into the near-Earth tail region. Note that the streamline remains on field lines that map to the LLBL while on the dayside. The second streamline starts along the noon-midnight meridian at the equator. The corresponding field lines are blue. Again the streamline enters the magnetosphere when the field line on which it is convecting reconnects with high latitude tail field lines. The streamline then moves along the dayside LLBL and into the tail.

The magnetospheric configuration changes significantly when the IMF has a $B_{Y}$ component. In the MHD model for purely northward IMF, the IMF field lines reconnect simultaneously in the northern and southern hemisphere forming closed field lines. When there is a $\mathrm{B}_{\mathrm{Y}}$ component the reconnection can occur in only one hemisphere. In Figure 3 we have plotted pressure contours in the equatorial plane along with selected magnetic field lines from a snapshot from a simulation when the IMF had northward and dawnward components. At this time the IMF had been northward for 45 minutes and duskward for 90 minutes. The pressure contours have been made transparent so that field lines (in purple) south of the equator can be seen. Note the open field lines shown here along the dusk flanks. A flow streamline starting in the southern hemisphere just duskward of noon has been drawn in orange. As in the purely northward IMF case, this streamline enters the magnetosphere at approximately position 2 when the field line on which it is convecting reconnects at high latitude. The streamline enters the magnetosphere on open field lines and remains on open field lines until it exits the simulation box. 
We also launched a distribution of solar wind test particles in the magnetic and electric fields from this MHD simulation. The distribution function of the test particles was selected to have the same plasma moments as the MHD simulation. The test particles were all protons and were launched in the plane at $x=20 \mathrm{R}_{\mathrm{E}}$. The white trace in Figure 3 gives the trajectory of one of these ions. This proton has energy of about $190 \mathrm{eV}$ in the solar wind frame. The ion entered the magnetosphere at approximately position 2. Again the entry mechanism is high latitude reconnection. Using similar calculations Richard et al., [1994] determined that high latitude reconnection is the main way in which ions enter the magnetosphere when the IMF is northward. After entering the magnetosphere the ion bounced several times at high southerly latitudes before moving onto open field lines and then into the polar cusp region. The ion emerged from the cusp onto closed dayside field lines. In Figure 4 this trajectory has been plotted from two perspectives. The view in the top panel is from the afternoon while the view in the bottom is from the morning. Field lines along the trajectory have been color coded to indicate the magnetic field magnitude. Note that soon after the ion crossed the magnetopause it bounced about the off equatorial minimum $|\vec{B}|$ that is characteristic of the magnetosphere just earthward of the dayside magnetopause. This type of oscillation about the high-latitude minimum $|\vec{B}|$ has been reported by Delcourt et al. [1992] based on particle calculations in empirical $\vec{E}$ and $\vec{B}$ field models (see their Figure 4). However, by the time the ion in Figure 4 reached the morning side (bottom) it was on a trapped orbit bouncing about the equator. In between the ion moved straight across the dayside cusp near the region where the high latitude reconnection is occurring. 
The effects of the motion across the cusp can be seen in Figure 5. Here the trajectory has been projected onto three orthogonal planes. Each trajectory is color coded to show the energy of the particle. While the ion is on the dusk side it has about the same energy as it had in the magnetosheath. However, when it moved across the polar cusp it quickly gained $\sim 20 \mathrm{kV}$. Richard et al., [1994] argued that ions move non-adiabatically in the polar cusp region when high latitude reconnection causes the radius of curvature of field lines there to become comparable with the ion gyro-radius. This non-adiabatic motion carries the ions across the high latitude electric field where they are accelerated.

The ion in Figure 5 is unusual in two ways. First it gained more energy in the cusp region than typical ions. Second it entered the cusp region after moving in the dusk side magnetosphere. However, our trajectory calculations indicate that non-adiabatic motion across the polar cusp region is the main way in which the most energetic ions are accelerated. Most of the accelerated ions enter the cusp directly from the magnetosheath when the field line on which they are moving reconnects at high latitudes.

In Figure 6 we have plotted the trajectory of an ion that did not enter the high latitude reconnection region. This particle entered the magnetosphere very close to noon when the field line on which it was moving reconnected with the southern lobe field (3). It quickly moved to closed field lines and bounced and drifted near the dayside magnetopause to the night side (4-6). In Figure 7 we have plotted the energy of this particle along its trajectory. This ion gained less than one $\mathrm{keV}$ as it moved across the dayside magnetosphere. 
Table 1

Ion Entry Rates $B_{\mathrm{Z}}>0$

\begin{tabular}{|c|c|c|}
\hline & MHD & Ion Trajectory \\
\hline$B_{Y}=0$ & $4 \times 10^{26} / \mathrm{s}$ & $1 \times 10^{27} / \mathrm{s}$ \\
\hline$B_{\mathrm{Y}} \neq 0$ & $5 \times 10^{26} / \mathrm{s}, 5 \times 10^{27} / \mathrm{s}$ & $2 \times 10^{27} / \mathrm{s}, 5 \times 10^{27} / \mathrm{s}$ \\
\hline
\end{tabular}

In Table 1 we have estimated the ion entry rates for the northward IMF simulations. We estimated the entry rate in the MHD simulation by determining the streamlines that cross the magnetopause onto magnetospheric field lines and calculating $\int \rho \vec{v} \cdot d \vec{s}$ where $\rho$ is the mass density, $\vec{v}$ is the fluid velocity and $d \vec{s}$ is an area element on the magnetopause. The integral was taken over the magnetopause area threaded by penetrating streamlines. Since the variable in the MHD simulations is the mass density, we assumed the solar wind to consist solely of protons in order to obtain the entry rate. When the IMF is purely northward $4 \times 10^{26} / \mathrm{s}$ enter on closed field lines. When the IMF has a $B_{Y}$ component we have listed two numbers. The first is the entry rate on closed field lines. It remains about the same as in the case with $B_{Y I M F}=0$. The second number is the entry rate on both open and closed field lines. It is an order of magnitude larger because of ions that are inside the magnetopause but on open field lines. For the $B_{Y} \neq 0$ case we used a simulation with $B_{Y}=-3.5 n T$ and $B_{Y}=3.5 n T$ that was run until a quasi-steady magnetosphere was formed (2 hours).

We also estimated the entry rate by using the particle trajectory calculations. The entry rate is given by $r=C\left(A \times v_{S W} \times n_{S W}\right) / N$ where $C$ is the number of ions entering the 
magnetosphere, $N$ is the total number launched, $A$ is the area in the solar wind over which the ions were launched, $v_{S W}$ is the solar wind velocity and $n_{S W}$ is the solar wind number density. In general the source rate estimates are larger from the trajectory calculations. In Figure 8 we have plotted the location on the magnetopause where the ions entered the magnetosphere. The entry points are bunched near the polar cusp. In MHD the comparable distribution has uniform entry. We get a higher estimate of the source rate from the particle calculations because of the enhanced entry in the cusp that is caused by the non-adiabatic motion [Richard et al., 1994].

\section{Southward IMF results}

We have plotted flow streamlines and pressure contours for a case with purely southward IMF in Figure 9. This snapshot was taken 60 minutes after the IMF was turned southward from northward. By this time subsolar point reconnection had been occurring for approximately 45 minutes [Walker et al., 1993; Bargatze et al., 1999]. The magnetosphere had not reached a steady state. A few minutes ( $t=70$ minutes) after this snapshot reconnection began in the near-Earth $\left(x \approx-15 R_{E}\right)$ magnetotail. About 10 minutes later reconnection began on lobe field lines. Since the magnetospheric configuration was not steady we calculated flow streamlines through a series of time steps starting before and extending after this time. Since the results were very similar to those in Figure 9 we have not included them in this paper.

In the simulation flow streamlines from the solar wind do not reach the inner parts of the magnetosphere. The streamlines that reach the middle and inner parts of the magnetosphere come from the inner boundary of the simulation. Streamlines in the LLBL come from both the solar wind and the inner boundary. Those nearest the Earth come 
from the inner boundary while streamlines nearer the magnetopause come from both sources. Note that the earthward flowing streamlines from the inner boundary turn around in the dayside magnetosphere and join with streamlines from the solar wind in flowing tailward. This can be seen more clearly in Figure 10. Here we have plotted only three streamlines along with magnetic field lines along the streamlines. The two sources of the LLBL have blue and green streamlines. Plasma on the blue streamlines enters the magnetosphere when the field line on which it is convecting reconnects at local times away from noon (2). This plasma moves on open field lines through the LLBL into the tail (3-5). In this example plasma from the inner boundary has taken a less direct path to the LLBL. The streamline emerges from the inner boundary on the dayside (green 1) and moves toward the dayside magnetopause. There it is picked up by reconnection (2) and moves on open field lines into the tail (3-5). In the tail it is picked up by earthward convection (6) and moves back toward the dayside magnetosphere (6-8). At the dayside magnetopause (between 8 and 9) the streamline again encounters reconnection. Finally it moves tailward on open field lines (10). In this example we followed a flow streamline from the inner boundary on the dayside. However plasma also emerges from the inner boundary on the nightside (Figure 9). These streamlines come from auroral latitudes. When the nightside flow streamlines reach the equator they too flow earthward into the dayside magnetosphere. Most of the outflow at the inner boundary is field aligned.

The red field lines in Figure 10 have been calculated along a streamline that originates in the solar wind along the Earth-Sun line. It too enters the magnetosphere because of dayside reconnection (2) but then moves on open field lines the length of the simulation box (3-5). 
We also launched a distribution of ions from the solar wind into the electric and magnetic fields from this simulation. We have plotted an example showing the trajectory of one of those particles in Figure 11. This ion was launched along the Earth-Sun line and had an initial energy of $17 \mathrm{eV}$ in the solar wind frame. This ion had a small southward $v_{\|}$.

Even in the solar wind the ion diverged slightly from the corresponding flow streamline. This became much more pronounced after it crossed the bow shock. The ion entered the magnetosphere when the field line on which it was moving reconnected (2). The ion moved along open field lines into the magnetotail (3-5) and reached the equator at $x \approx-50 R_{E}(6)$. Then it drifted earthward (7) and around the Earth before exiting the magnetosphere. By location (8) the field lines on which the ion was moving were in the LLBL very near the magnetopause. Finally the ion left the magnetosphere when the field line on which it was moving reconnected.

Table 2

Ion Entry Rates $B_{Z}<0$

\begin{tabular}{|c|c|c|}
\hline & MHD & Ion Trajectory \\
\hline $\begin{array}{c}\text { Solar Wind- } \\
\text { Open Field Lines }\end{array}$ & $1 \times 10^{28} / \mathrm{s}$ & $2 \times 10^{28} / \mathrm{s}$ \\
\hline $\begin{array}{c}\text { Solar Wind - } \\
\text { Closed Field Lines }\end{array}$ & & $2 \times 10^{27} / \mathrm{s}$ \\
\hline Solar Wind - Direct Entry & & \\
\hline Ionosphere & $3 \times 10^{26} / \mathrm{s}$ & $<1 \times 10^{26} / \mathrm{s}$ \\
\hline
\end{tabular}


We also estimated the ion source rate for this simulation. The results are summarized in Table 2. We calculated the solar wind source rate using the same method we used for the northward IMF case. In the MHD calculation about $1 \times 10^{28} / \mathrm{s}$ solar wind ions were entering the magnetosphere on open field lines at 60 minutes. As noted above this plasma exited the magnetotail boundary of the simulation without entering the closed field line region. That region of the magnetosphere was populated from the inner boundary of the simulation. We calculated the ionospheric source rate by integrating $\int \rho \vec{v} \cdot d \vec{s}$ over a closed surface at the inner boundary. This gave $3 \times 10^{26} / s$ if we assume protons and $2 \times 10^{25} / s$ if $\mathrm{O}^{+}$. From the trajectory calculation we estimate that $2 \times 10^{28} / \mathrm{s}$ solar wind ions enter on open field lines but that only about one in ten reach closed field lines. Finally a very few ions enter the magnetosphere by drifting through the magnetopause. Since the number of these particles is very low we could only obtain an upper limit of $1 \times 10^{26} / s$ for this source.

\section{Discussion}

In all of our calculations solar wind ions primarily enter the magnetosphere when the field line on which they are convecting reconnects at the dayside magnetopause. For northward IMF the reconnection is in the polar cusp and these particles form the low latitude boundary layer. For $B_{Z}>0$ and $B_{Y} \neq 0$ these ions can be on either open or closed field lines. Open field lines occur when the high latitude reconnection occurs in only one cusp. Since cases with simultaneous or nearly simultaneous reconnection in both cusps are thought to be rare [Fuselier et al., 2001], this is probably the way LLBL ions usually enter the magnetosphere. These ions quickly move from open to closed field lines. In the 
MHD simulation the ionosphere does not contribute significantly to the LLBL for northward IMF.

The situation is much more complex for southward IMF. In the MHD calculation solar wind plasma enters the magnetosphere on reconnecting field lines but never makes it to the closed field line region. Instead the open field lines convect to the end of the simulation box. Along the flanks of the magnetosphere open field lines carrying solar wind plasma are found in the same region as open field lines carrying ionospheric plasma. This region forms the outer part of the LLBL. Nearer the Earth the plasma in the MHD simulation is dominated by ionospheric plasma. When closed field lines carrying this plasma approach the magnetopause they can reconnect adding the ionospheric plasma to the LLBL mix. In the MHD simulation the inner regions of the LLBL are on closed field lines containing this ionospheric plasma. However, when we include the particle trajectory results we find that solar wind plasmas can reach the closed field region. Thus for southward IMF we would expect the LLBL to contain plasmas of both ionospheric and solar wind origin.

Very recently Siscoe et al., [2001] have examined flow streamlines from an MHD simulation with $B_{Z}<0$. They too found that solar wind plasma on reconnected field lines passes through the back of the simulation box (compare their Figure 5 with Figure 9 (top)). In their simulation the plasma from the cusp region fills the plasma mantle and outer plasma sheet. They call the boundary between these two types of behavior the "fluopause". The "cusp" plasma in their simulation comes from the ionospheric boundary much like it does in our simulation (compare their Figure 7 with Figure 9 (bottom)). The "cusp" particles in both simulations populate the more distant parts of the tail. In our 
calculation we continued to follow the streamlines throughout the magnetosphere and found that those in the middle tail return toward the Earth and end up populating the LLBL as well. We also found that the inner (ionospheric) boundary provides the plasma for the near magnetotail. This plasma comes from the nightside ionosphere boundary (Figure 9 top). Siscoe et al., [2001] did not present streamlines in this region. Overall the agreement between the two simulations is excellent. It is reassuring when two codes using very different approaches get the same results.

Winglee [1998; 2000] has developed a multifluid MHD simulation that includes ionospheric outflow as a separate fluid. He too reports that the ionospheric plasma is confined to the inner magnetosphere for northward IMF while for southward IMF ionospheric plasma overcomes gravity and fills all but the more distant parts of the tail.

Peroomian [2001] has mapped the locations where solar wind ions reach the magnetopause by calculating ion trajectories in a global MHD model. He used a somewhat stronger solar wind and found a few times $10^{28} / \mathrm{s}$ reached the magnetopause and that more ions reached the magnetopause for purely southward IMF than when there was an IMF $B_{Y}$. He did not calculate the number entering the magnetosphere so we cannot compare the population of various regions of the magnetosphere.

The source rates from the simulations in Tables 1 and 2 are in reasonable agreement with inferences from observations [Hultqvist et al., 1999]. When we use the trajectory calculations to estimate the solar wind source rates we get consistently higher source rates than with the MHD simulations. The largest difference occurs when the IMF is northward. This is primarily the result of entry into the polar cusp. Ions can undergo non-adiabatic acceleration in the cusp regions. In our simulations ions gained up to 
$20 \mathrm{keV}$ but acceleration to a few $\mathrm{keV}$ is more common. Finally we should remind the reader that the particle trajectory calculations are not self-consistent. The particles' motion cannot influence the electric and magnetic fields through which they move. In reality the fields will change and so will the trajectories. Thus the trajectory plots shown in this paper should be thought of as a simple approximation to the actual motion.

Moore et al., [1999] have summarized the ionospheric outflow to the magnetosphere based on observations from polar orbiting satellites (see their Table 2.1). They find outflows from $1 \times 10^{25} / \mathrm{s}-1 \times 10^{26} / \mathrm{s}$ for $\mathrm{H}^{+}$and $2 \times 10^{25} / \mathrm{s}-2 \times 10^{26} / \mathrm{s}$ for $\mathrm{O}^{+}$with larger values for active times at solar maximum. The values in Table 2 fall at the low end of this range. The low energy $(<100 \mathrm{eV})$ observations used by Moore et al., [1999] come from low altitude $(6000-10,000 \mathrm{~km})$ spacecraft while the high energy observations $(10 \mathrm{eV}$ $17 \mathrm{keV})$ are from high altitudes $(16,000-24,000 \mathrm{~km})$. Since the low energy ions can be energized as they move upwards the observations may be over estimates. In our calculations the ionospheric source is passive. While we do not specify any ionospheric outflow the ionospheric boundary condition provides a cache of plasma that can be moved outward. The ionospheric outflow results solely from the solar wind magnetosphere interaction in our calculation.

We have not carried out calculations in which we launch ionospheric ions in the global MHD fields. Other [Delcourt et al., 1992; 1994; Peroomian and Ashour-Abdalla, 1996] have followed the trajectories of ionospheric ions in empirically based electric and magnetic field models. Delcourt et al., [1992; 1994] have followed protons from the tailward edge of the polar cusp into the magnetotail. The trajectories of their lowest energy particles $(<100 \mathrm{eV})$ are very much like the tailward part of the ionospheric 
streamlines in Figure 9. Peroomian and Ashour-Abdalla [1996] launched $\mathrm{H}^{+}, \mathrm{He}^{+}$, and $\mathrm{O}^{+}$ ions from the auroral zone. These ions populated the near-Earth tail region and the dayside magnetosphere. In the MHD model the auroral zone streamlines in Figure 9 (top) cover approximately the same region in the tail but don't extend to the magnetopause on the dayside.

Whether the IMF is northward or southward the simulation results indicate that reconnection is responsible for most plasma entry into the magnetosphere. While few would argue that reconnection is the dominate mechanism driving magnetospheric dynamics since its discovery [Hones et al.,1972; Eastman et al., 1976] it has been tempting to associate the LLBL with a viscous component of the solar wind magnetosphere interaction as well. Suggestions for the viscous stress include unstable Kelvin-Helmholtz (K-H) waves [Lee et al., 1981; Pu and Kivelson, 1983] that in principle can be studied with global MHD simulations. Two dimensional MHD models have been very successful in delimiting the flow and plasma conditions under which the K-H instability can grow on the dayside magnetopause [Muira, 1995] and along the flanks of the magnetotail [Otto and Fairfield, 2000]. In the Otto and Fairfield [2000] study in the nonlinear stage of the $\mathrm{K}-\mathrm{H}$ vortices, the magnetic field becomes highly twisted and local regions of oppositely directed field develop at which local reconnection allows plasma mixing across the magnetopause. To our knowledge only one 3D global MHD model has reported K-H waves. Walker et al., [1998] modeled the magnetosphere under conditions designed to maximize the chance of generating the K-H instability. They used a global MHD code to simulate the response of the magnetosphere to a density pulse (the density increased from $46 \mathrm{~cm}^{-3}$ to $185 \mathrm{~cm}^{-3}$ and back to $46 \mathrm{~cm}^{-3}$ ) when the IMF 
was strongly northward $\left(B_{z}=15 n T\right)$. Since the wavelengths of the K-H waves are on the order of the thickness of the LLBL (as small as $0.5 R_{E}$ ) a very fine grid was used $\left(\Delta x=0.15 R_{E}\right)$. The strong northward IMF was selected since Muira [1995] has shown that southward IMF stabilized the boundary and the density was increased to lower the Alfvén velocity. After the pulse passed the magnetopause small K-H like flow vorticies were found along the flank magnetopause. We have examined the simulation in Figure 1 for evidence of $\mathrm{K}-\mathrm{H}$ waves but did not find any. Additional runs with higher resolution and varying solar wind parameters will be needed before we can use the simulation results to place limits on $\mathrm{K}-\mathrm{H}$ waves as a source of LLBL plasma.

Acknowledgements. The work at UCLA was supported by the NASA International Solar Terrestrial Physics Program grant NAG5-6689. The work at Nagoya University was supported by grants in aid from the Ministry of Education, Science and Culture.

Computing support was provided by the Computer Center of Nagoya University and by the National Partnership for Supercomputing Infrastructure at the San Diego Supercomputer Center.

\section{References}

Abe, T., S. Watanabe, B. A. Whalen, A. W. Yau, and E. Sagawa, Observations of polar wind and thermal ion outflow by Akebono/SMS, J. Geomag. Geoelectr., 48, 319, 1996.

Ashour-Abdalla, M., J. Berchem, J. Buchner, L. M. Zelenyi, Shaping of the magnetotail from the mantle: Global and local structuring, J. Geophys. Res., 98, 5651, 1993.

Bargatze, L., F., , T. Ogino, R. L., McPherron, and R. J. Walker, Solar wind magnetic field control of magnetospheric response delay and expansion phase onset timing, J. Geophys. Res., 104, A7, 14583, 1999.

Delcourt, D. C., T. E. Moore, J. A. Sauvaud, and C. R. Chappell, Nonadiabatic transport features in outer cusp region, J. Geophys. Res., 97, A11, 16,833, 1992.

Delcourt, D. C., T. E. Moore and C. R. Chappell, Contribution of low-energy ionospheric protons to the plasma sheet, J. Geophys. Res., 99, A4, 5681, 1994. 
Eastman, T. E., E. W. Hones, Jr., S. J. Bame, and J. R. Ashbridge, The magnetospheric boundary layer: Site of plasma, momentum and energy transfer from the magnetosheath into the magnetosphere, Geophys. Res. Lett., 3, 685, 1976.

Fusilier, S., A., S. M. Petrinec, K. J. Trattner, W. K. Peterson, $\mathrm{O}^{+}$observations in the cusp: implications for dayside magnetic field topology, J. Geophys. Res., 106, A4, 5977.

Hones, E. W., Jr., J. R. Ashbridge, S. J. Bame, M. D. Montgomery, S. Singer, and S.-I. Akasofu, Measurements of magnetotail plasma flow made with Vela 4B, $J$. Geophys. Res., 77, 5503, 1972.

Hultqvist, B., M. Andre, S. P Christon, G. Paschmann, D. G. Sibeck, Contributions of different source and loss processes to the plasma content of the magnetosphere, Space Science Rev., 88,1-2, 355, 1999.

Lee, L.C., R. K. Albano, and J. R. Kan, Kelvin-Helmholtz instability in the magnetopause-boundary layer region, J. Geophys. Res., 86, A1, 54, 1981.

Moore, T. E., R. Lundin, D. Alcayde, M. Andre, S. B. Ganguli, M. Temerin, and A. Yau, Source processes in the high-latitude ionosphere, Space Science Rev., 88,1-2, 7, 1999.

Miura, A., Kelvin-Helmholtz instability at the magnetopause: Computer simulations, Physics of the Magnetopause, P. Song, B. U. O. Sonnerup, and M. F. Thomsen, ed., Geophys. Monograph, 90, 285, 1995.

Newell, P. T. and C.-I. Meng, Mapping the dayside ionosphere to the magnetosphere according to particle precipitation characteristics, Geophys. Res., Lett., 19, 609, 1992.

Ogino, T., R. J. Walker, and M. Ashour-Abdalla, A global magnetohydrodynamic simulation of the magnetosheath and magnetosphere when the interplanetary magnetic field is northward, IEEE Transactions on Plasma Science, 20, 817, 1992.

Ogino, T., R. J. Walker, and M. Ashour-Abdalla, A global magnetohydrodynamic simulation of the response of the magnetosphere to a northward turning of the interplanetary magnetic field, J. Geophys. Res., 99, 11027, 1994.

Otto, A., and D. H. Fairfield, Kelvin-Helmholtz instability at the magnetotail boundary: MHD simulation and comparison with Geotail observations, J. Geophys. Res., 105, A9, 21175, 2000.

Peroomian, V., Interplanetary magnetic field-dependent impact of solar wind ions on Earth's magnetosphere, (submitted) Geophys. Monograph, 2001.

Peroomian, V., and M. Ashour-Abdalla, Population of the near-Earth magnetotail from the auroral zone, J. Geophys. REs., 101, A7, 15,387, 1996.

$\mathrm{Pu}, \mathrm{Z} .-\mathrm{Y}$., and M. G. Kivelson, Kelvin-Helmholtz instability at the magnetopause: solution for compressible plasmas, J. Geophys. Res., 88, A2, 841, 1983.

Richard, R. L., R. J. Walker, and M. Ashour-Abdalla, The population of the magnetosphere by solar wind ions when the interplanetary magnetic field is northward, Geophys. Res., Lett., 21, 2455, 1994.

Sibeck, D. G., G. Paschmann, R. A. Treumann, S. A. Fuselier, W. Lennartsson, M Lockwood, R. Lundin, K. W. Olgvie, T. G. Onsager, T.-D. Phan, M. Roth, M. Scholer, N. Sckopke, K. Stasiewicz, and M. Yamauchi, Plasma transfer processes at the magnetopause, Space Science Rev., 88,1-2, 207, 1999. 
Siscoe, G. L., G. M. Erickson, B. U. Ö. Sonnerup, N. C. Maynard, K. D. Siebert, D. R. Weimer, and W. W. White, Relation between cusp and mantle in MHD simulation, J. Geophys. Res., 106, A6, 10,743, 2001.

Walker, R. J., T. Ogino, J. Raeder, and M. Ashour-Abdalla, A global magnetohydrodynamic simulation of the magnetosphere when the interplanetary magnetic field is southward: The onset of magnetotail reconnection, J. Geophys. Res., 98, 17,235, 1993.

Walker, R. J., T. Ogino, and M. Ashour-Abdalla, The response of the magnetosphere to a solar wind density pulse, Substorm 4, S. Kokubun and Y. Kamide, eds., Terra Scientific Publishing Company/ Kluwer Academic Publishers, Tokyo, 527, 1998.

Walker, R. J., R. L. Richard, T. Ogino, and M. Ashour-Abdalla, The response of the magnetotail to changes in the IMF orientation: The magnetotail's long memory, Physics and Chemistry of the Earth, 24, 221, 1999.

Winglee, R., Multi-fluid simulations of the magnetosphere: The identification of the geopause and its variation with IMF, Geophys. Res., Lett., 25, 24, 4441, 1998.

Winglee, R., Mapping of ionospheric outflows into the magnetosphere for varying IMF conditions, J. Atm. and Solar-Terrestrial Phys., 62, 6, 527, 2000.

Yau, A. W., E. G. Shelley, W. K. Peterson, and Lenchyshyn, Energetic auroral and polar ion outflow at DE-1 altitudes: magnitude, composition, magnetic activity dependence and long-term variations, J. Geophys. Res., 90, 8417, 1985. 


\section{Figures}
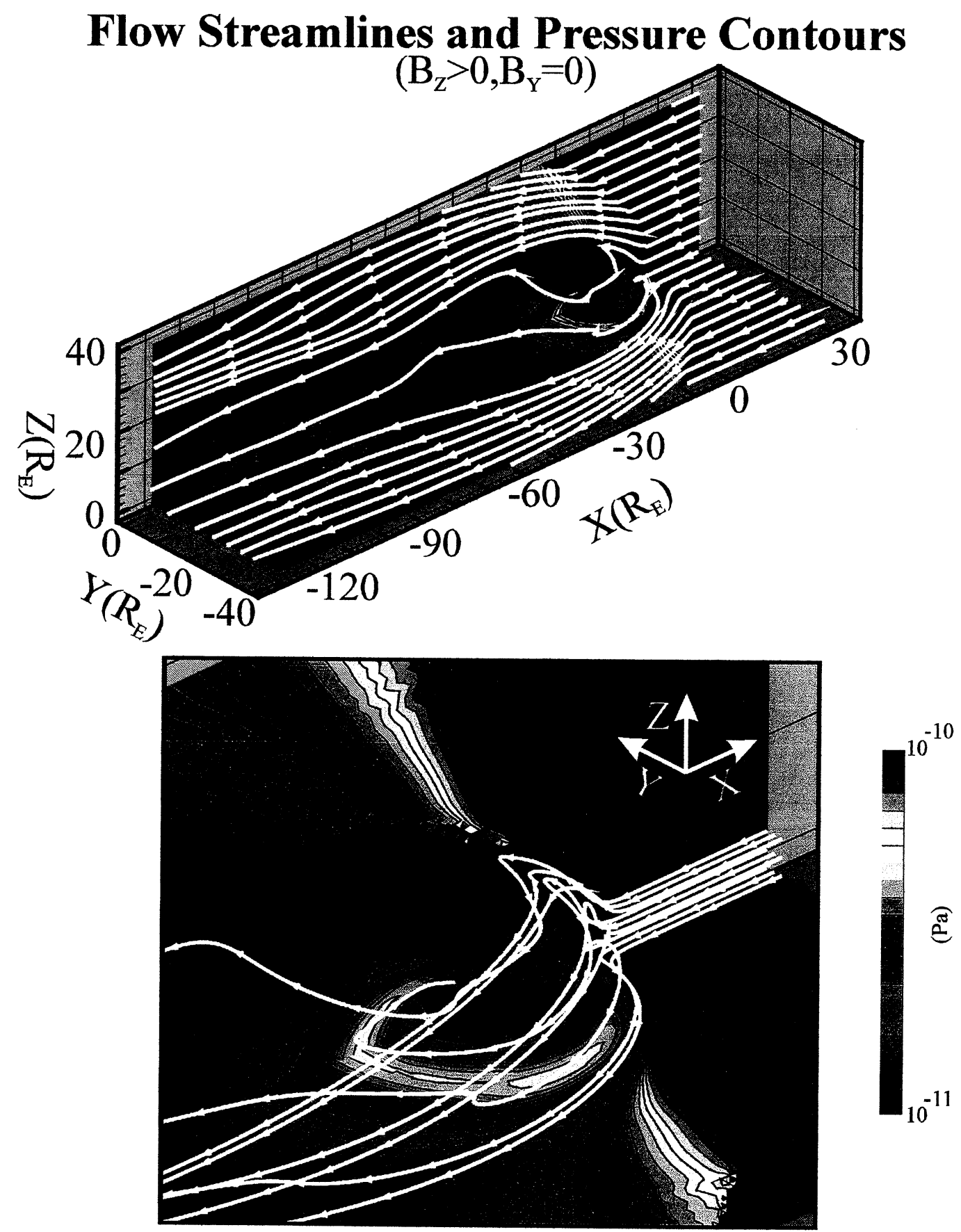

Figure 1. Flow streamlines and pressure contours for a simulation with IMF $B_{Z}>0$. The pressure contours were placed in the noon-midnight and equatorial planes. The flow streamlines were started in the solar wind. The bottom panel shows an enlargement of the region close to the Earth. Additional streamlines have been added near the Sun-Earth line. 


\section{Flow Streamlines and Magnetic Field Lines$$
\left(\mathrm{B}_{\mathrm{Z}}>0, \mathrm{~B}_{\mathrm{Y}}=0\right)
$$

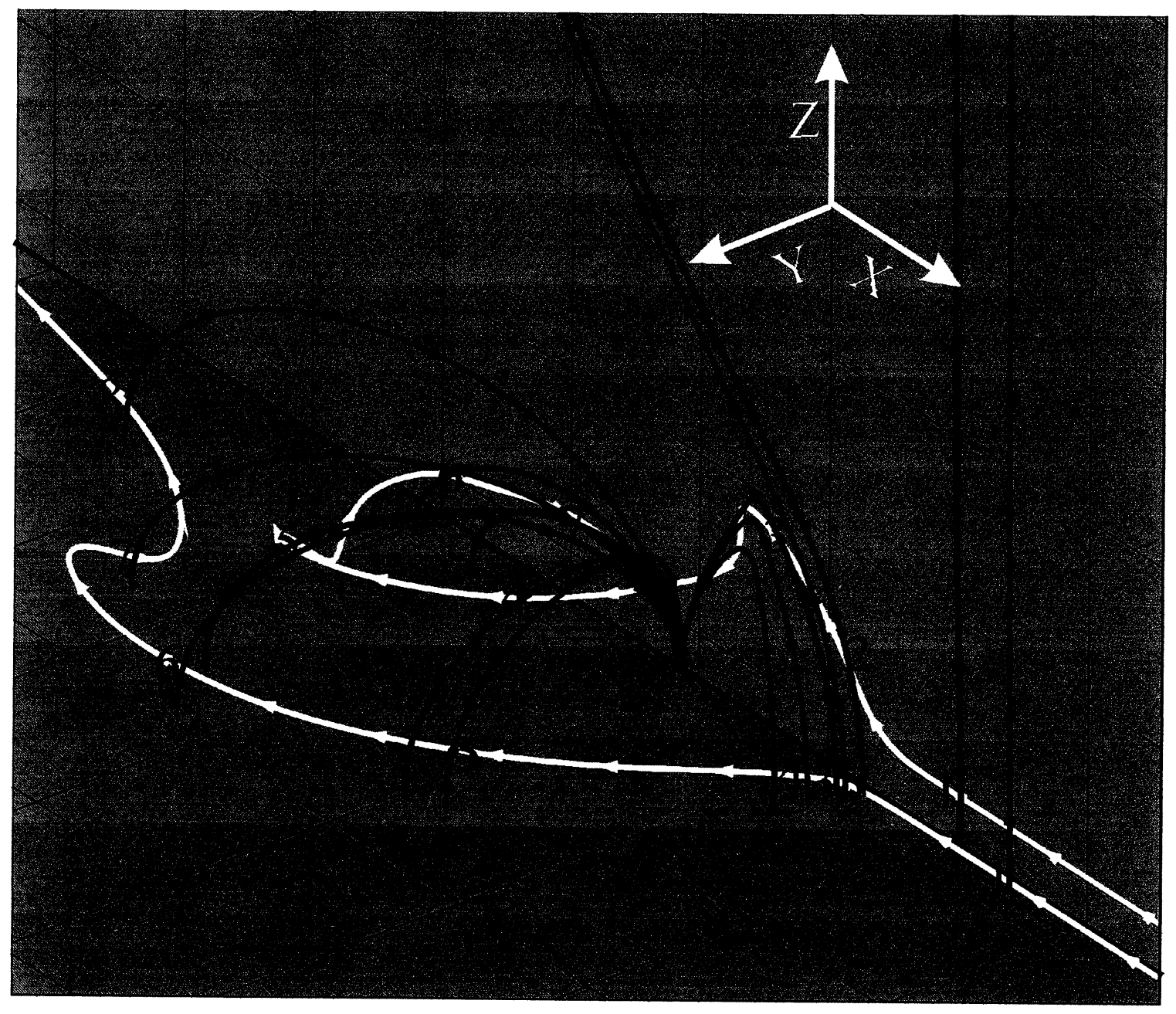

Figure 2. Magnetic field lines calculated along two flow streamlines from the simulation in Figure 1. The colored numbers indicate the locations along the streamlines discussed in the text. 


\section{Field Lines Along a Dusk LLBL Ion Trajectory

$$
\left(\mathrm{B}_{\mathrm{Z}}>0 ; \mathrm{B}_{\mathrm{Y}}>0\right)
$$

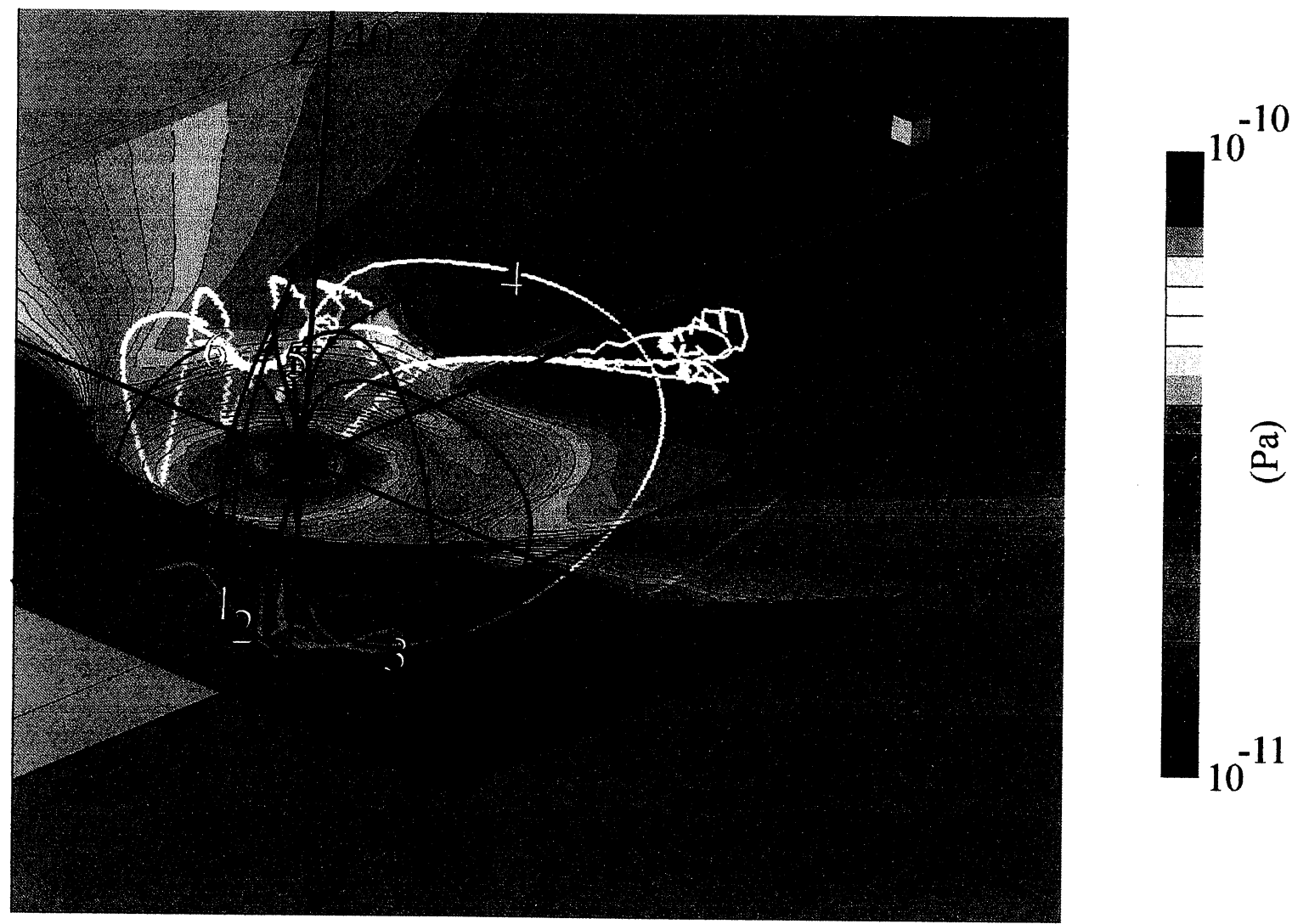

Figure 3. Field lines (purple) along the trajectory of a solar wind ion (white). The trajectory was calculated in the electric and magnetic fields from a simulation with IMF $B_{Z}>0$ and $B_{Y}>0$. At this time $B_{Z}=4.8 n T$ and $B_{Y}=1.3 n T$. Pressure contours have been plotted in the equatorial plane. They have been made semi-transparent. The orange line is a flow streamline started from the same position as the ion trajectory. 


\section{Field Lines Along a Dusk LLBL Ion Trajectory $\left(\mathrm{B}_{\mathrm{Z}}>0 ; \mathrm{B}_{\mathrm{Y}}>0\right)$}
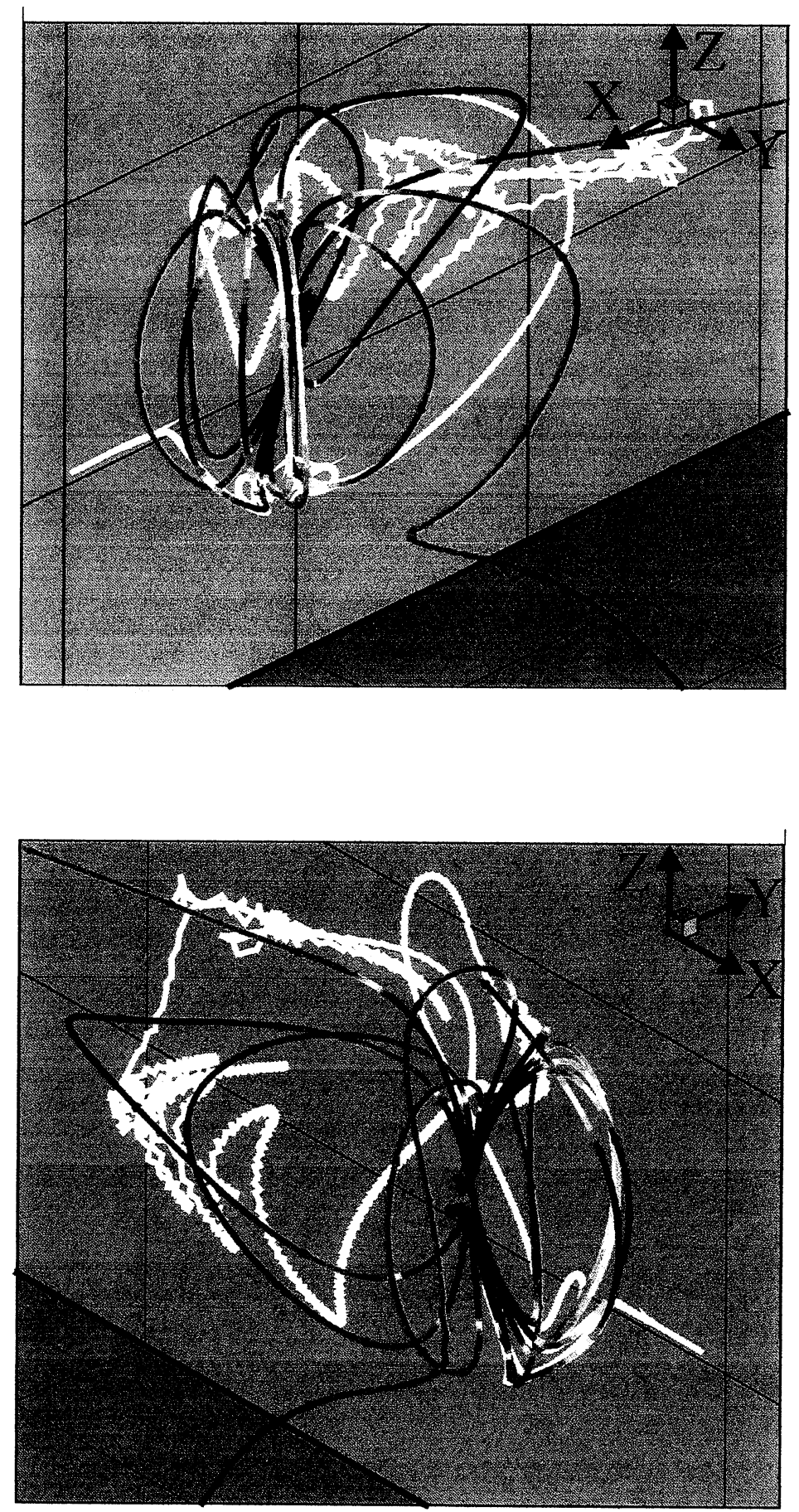

Figure 4. Two views of the ion trajectory in Figure 3. The view in the top panel is from the dusk side while the view in the bottom panel is from the dawn side. Magnetic field lines have been calculated along the trajectory. The color coding on the field lines gives the magnitude of the magnetic field. 


\section{Ion Energy Along Trajectory}

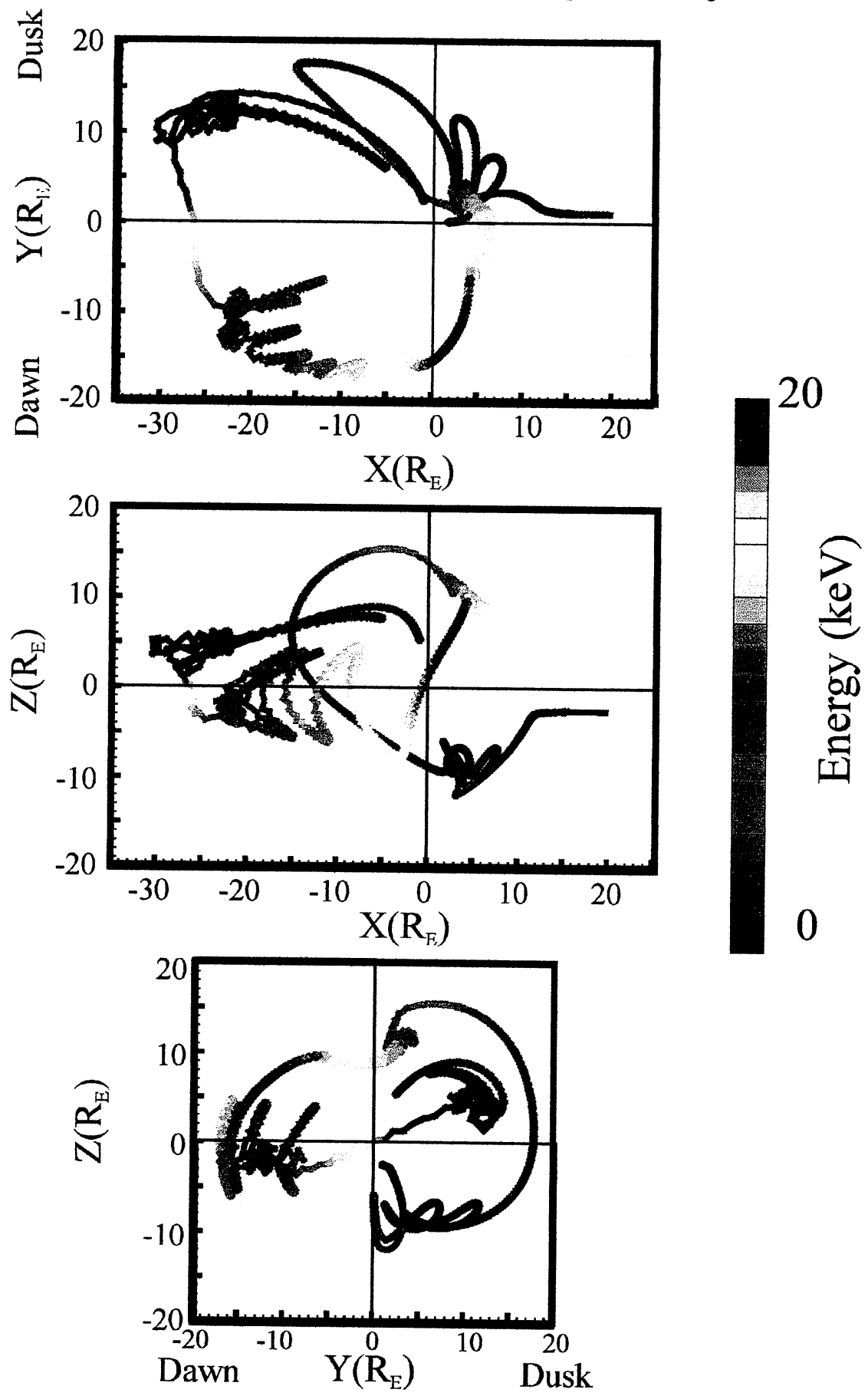

Figure 5. Projections of the ion trajectory in Figure 3 onto three planes $(Z=0, Y=0, X=0)$. The trajectory is color coded with the particle energy. 


\section{Field Lines Along an Ion Trajectory \\ $\left(\mathrm{B}_{z}>0 ; \mathrm{B}_{\mathrm{y}}>0\right)$}

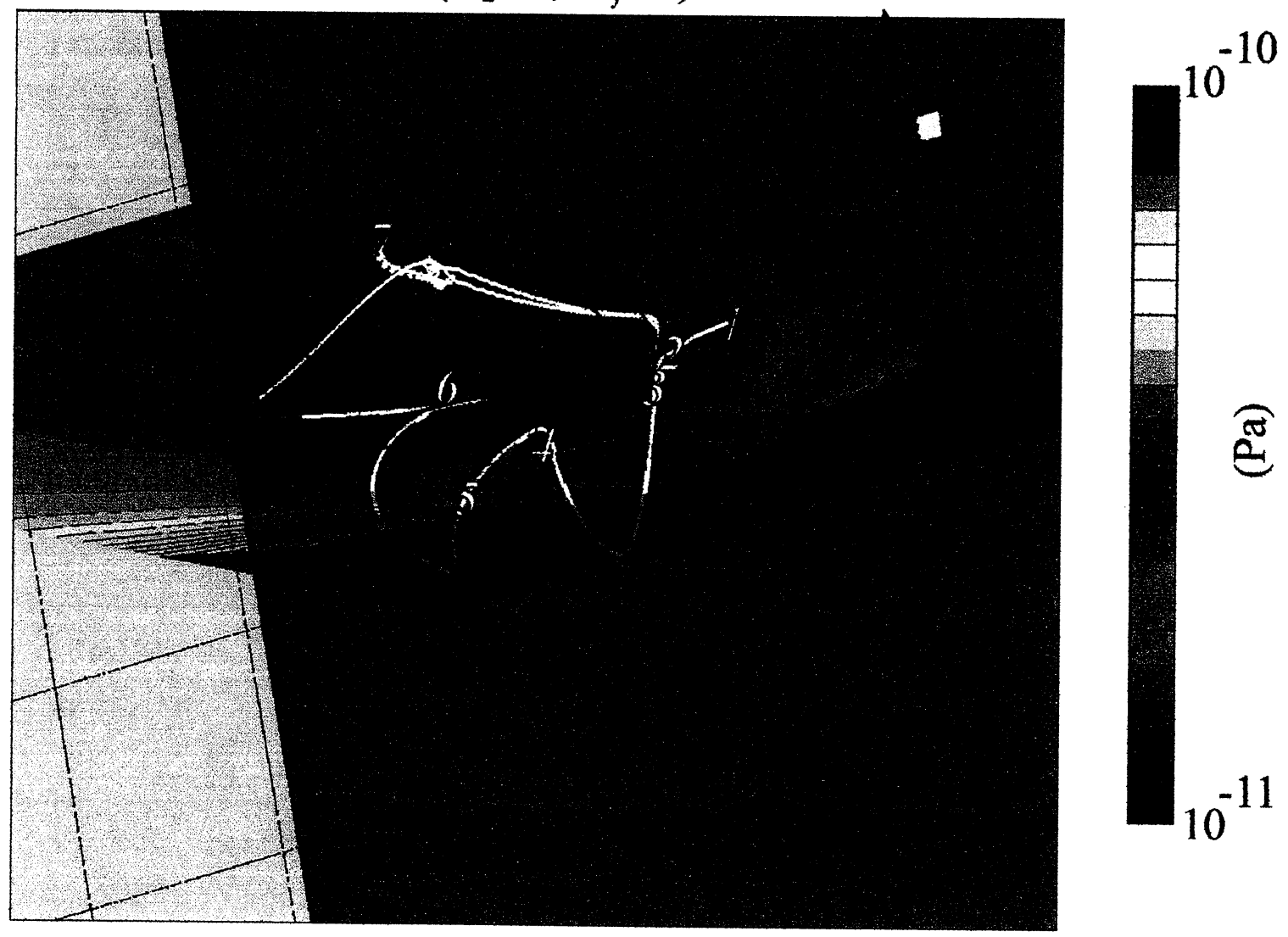

Figure 6. Field lines (purple) along the trajectory of a solar wind ion (white). The trajectory was calculated in the electric and magnetic fields from the same simulation as Figure 3. Pressure contours have been plotted in the equatorial plane. They have been made semi-transparent. The orange line is a flow streamline started from the same position as the ion trajectory. 


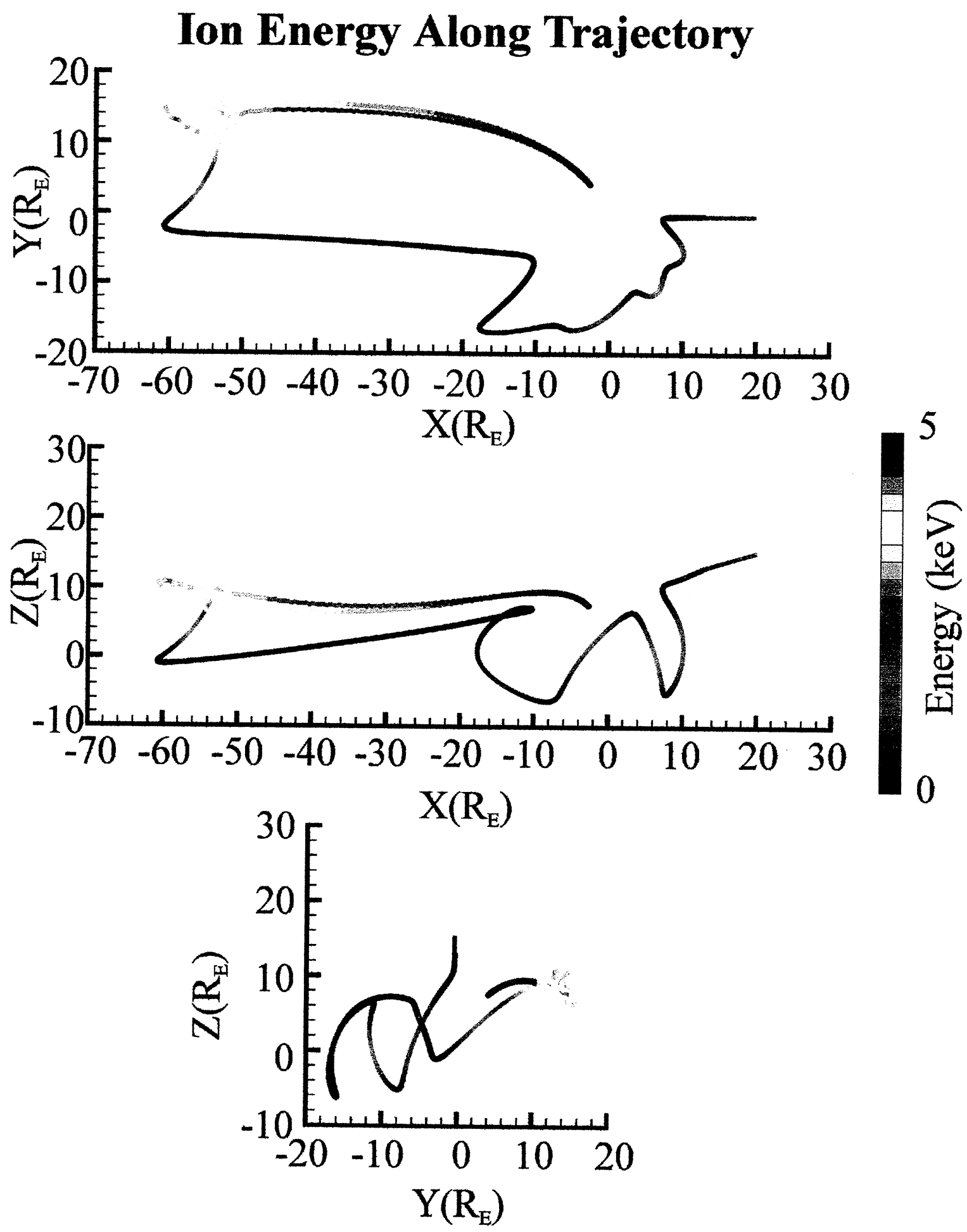

Figure 7. Projections of the ion trajectory in Figure 6 onto three planes $(Z=0, Y=0, X=0)$. The trajectory is color coded with the particle energy. 


\section{Ion Entry Locations}
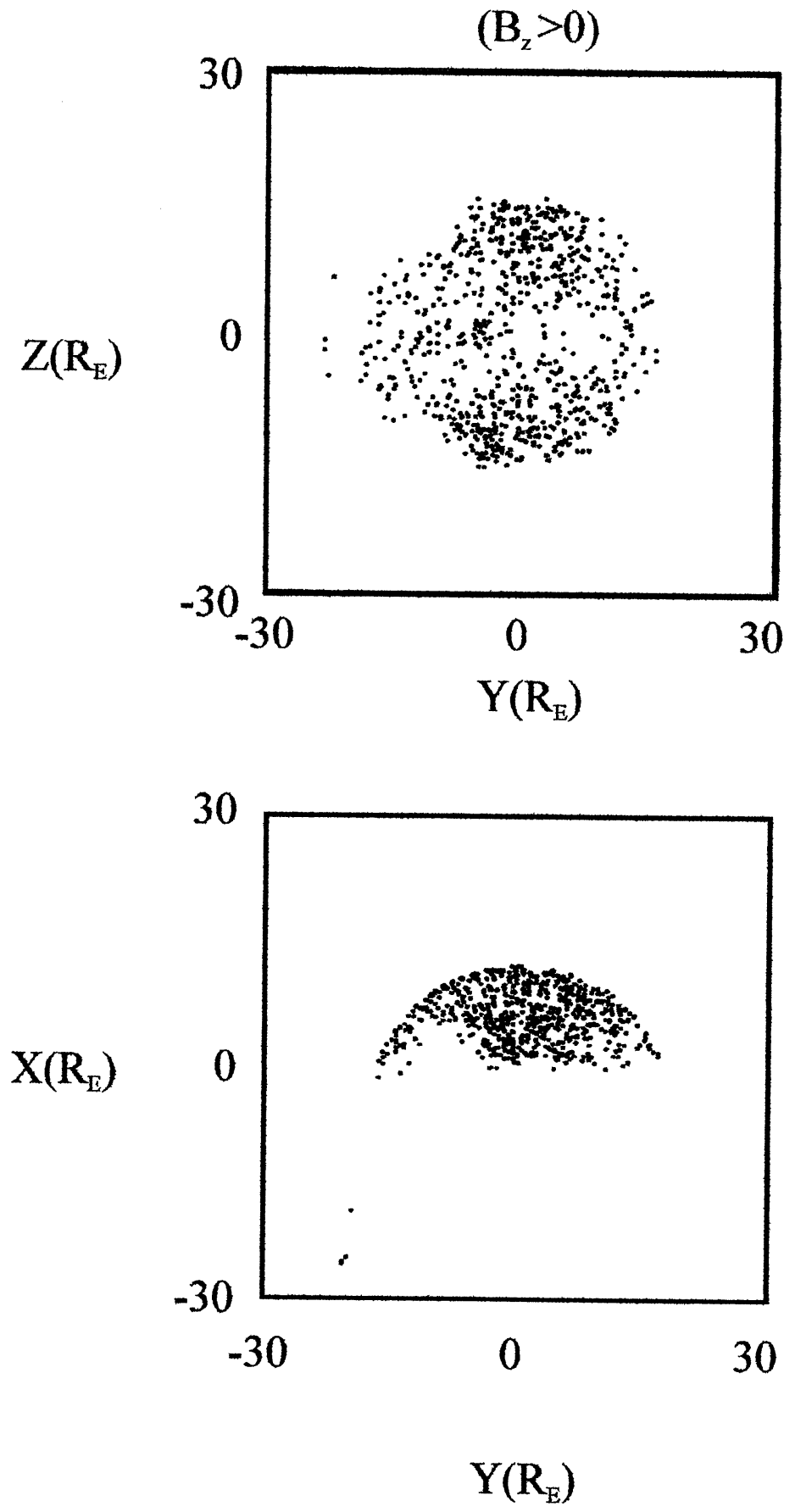

Figure 8. The distribution of ion entry locations on the dayside magnetopause. The top panel shows the entry locations projected onto the $\mathrm{YZ}$ plane while the bottom panel shows the locations projected onto the XY plane. The MHD simulation had IMF $B_{Z}>0$. (From Richard et al., 1994]. 


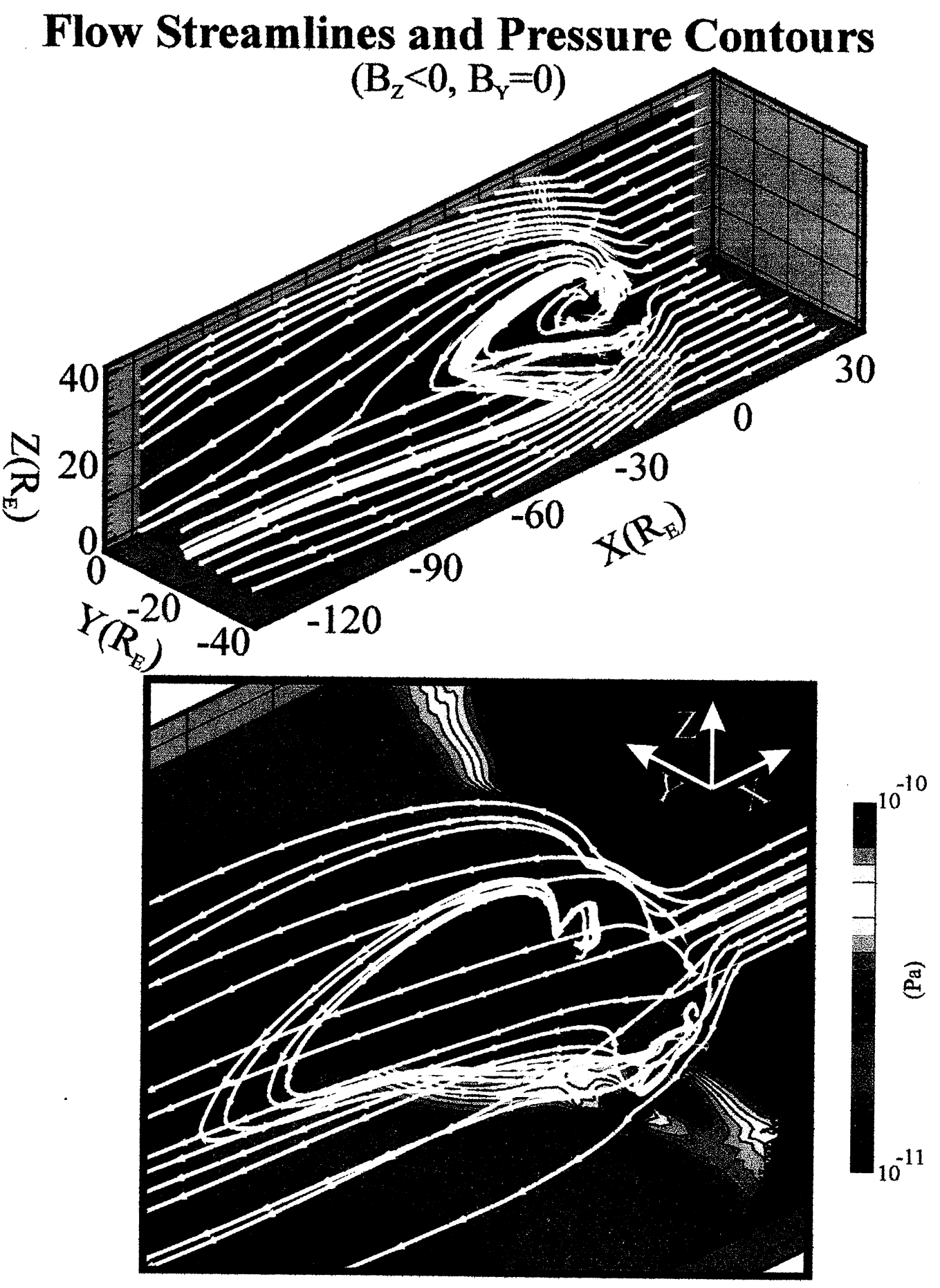

Figure 9. Flow streamlines and pressure contours for a simulation with IMF $\mathbf{B}_{Z}<0$. The pressure contours were placed in the noon-midnight and equatorial planes. The flow streamlines were started in the solar wind and in the middle and inner magnetosphere. The bottom panel shows an enlargement of the region close to the Earth. Additional streamlines have been added near the Sun-Earth line. 


\section{Flow Streamlines and Magnetic Field Lines $\left(\mathrm{B}_{\mathrm{Z}}<0, \mathrm{~B}_{\mathrm{Y}}=0\right)$}

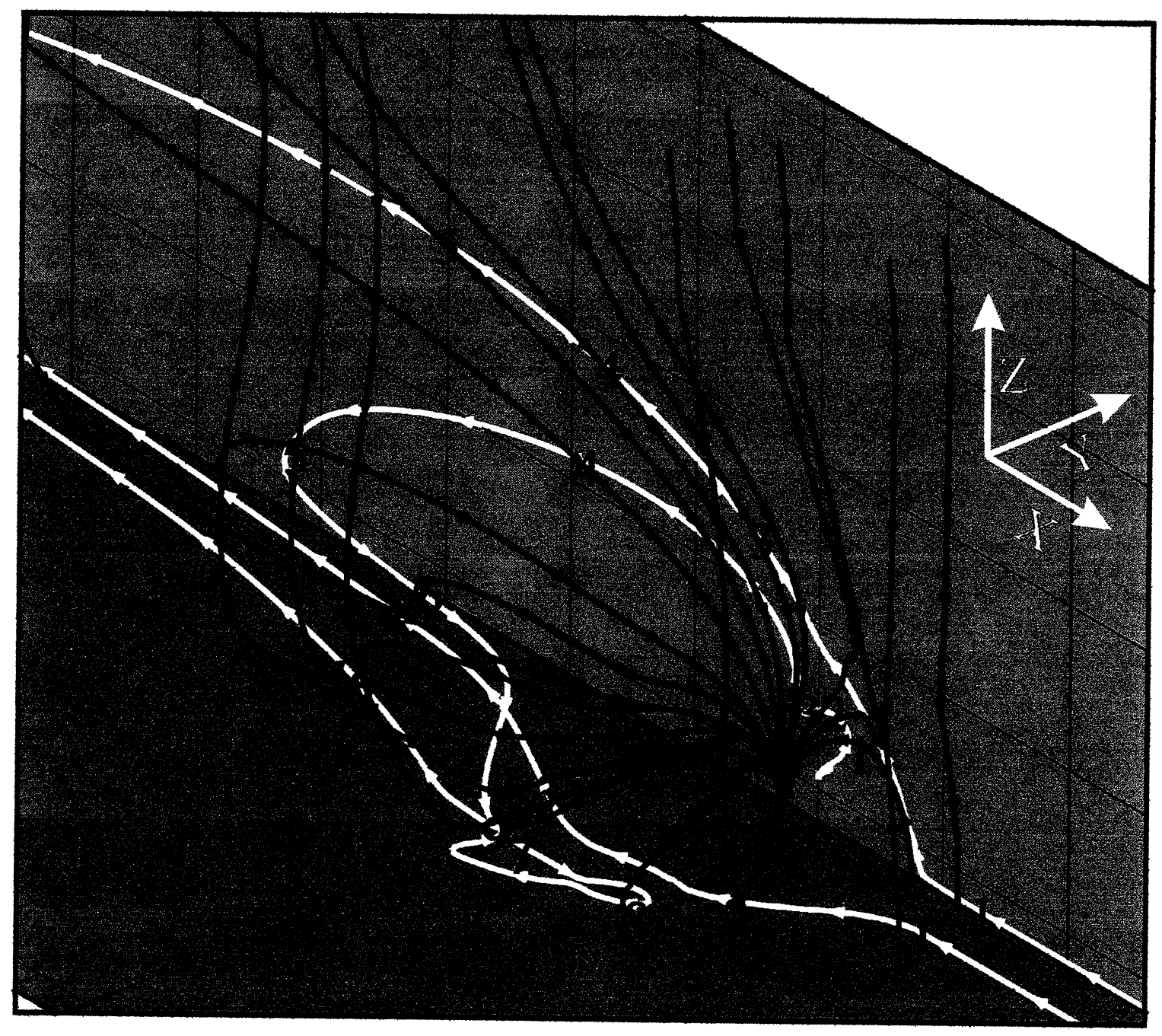

Figure 10. Magnetic field lines calculated along three flow streamlines from the simulation in Figure 9. The colored numbers indicate the locations along the streamlines discussed in the text. 


\section{Magnetic Field Lines Along an Ion Trajectory}

$$
\left(\mathrm{B}_{\mathrm{Z}}<0, \mathrm{~B}_{\mathrm{Y}}=0\right)
$$

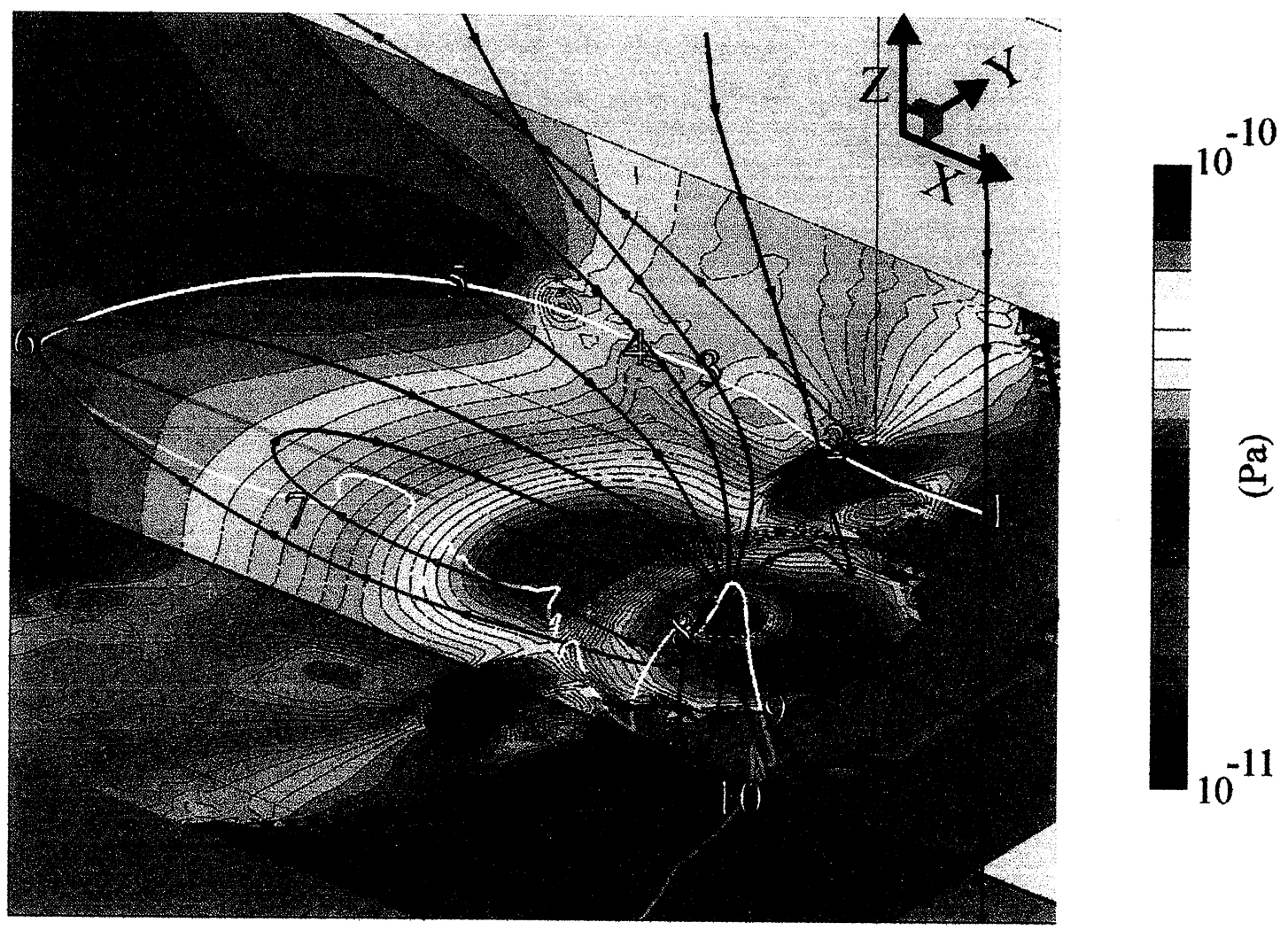

Figure 11. Field lines (purple) along the trajectory of a solar wind ion (white). The trajectory was calculated in the electric and magnetic fields from a simulation with the IMF $B_{Z}<0$. Pressure contours have been plotted in the equatorial plane. They have been made semi-transparent. The orange line is a flow streamline started from the same position as the ion trajectory. 